\title{
When disks collide: major and minor mergers in disk galaxies
}

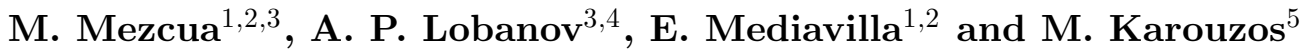 \\ ${ }^{1}$ Instituto de Astrofísica de Canarias (IAC), E-38200 La Laguna, Tenerife, Spain \\ email: mmezcua@iac.es \\ ${ }^{2}$ Universidad de La Laguna, Dept. Astrofísica, E-38206 La Laguna, Tenerife, Spain \\ ${ }^{3}$ Max Planck Institute for Radio Astronomy, Auf dem Hügel 69, D-53121 Bonn, Germany \\ ${ }^{4}$ Institut für Experimentalphysik, Universität Hamburg, \\ Luruper Chausse 149, D-22761 Hamburg, Germany \\ ${ }^{5}$ Center for the Exploration of the Origin of the Universe, Seoul National University, \\ Seoul 151-742, Korea
}

\begin{abstract}
We present the surface brightness profile fitting of a sample of double nucleus disk galaxies, minor merger candidates. We have decomposed these systems into two compact nuclear components and one or two extended galaxy disk components and estimated the luminosity of the primary and secondary nucleus and of the host galaxy and the separation between the two nuclei. Based on the ratio of nuclear luminosities we find that most of the sources qualify as major mergers despite their initial classification as minor merging systems. This is supported by the finding that $65 \%$ of the host galaxies are fitted only by one galaxy disk and that the luminosity of both the primary and the secondary nucleus decreases with decreasing nuclear separation, as expected from simulations of disk galaxy mergers. All these results indicate that these sources are most plausibly in the post-merger state of a major merger event. We also identify 19 candidates to binary active nucleus with nuclear separation $\leqslant 1 \mathrm{kpc}$.
\end{abstract}

Keywords. Galaxies: nuclei - interactions - photometry - structure

\section{Introduction}

In the hierarchical galaxy formation model in a a $\Lambda$ CDM Universe, major mergers (with mass ratio $>1: 3$ ) dominate the formation of massive bulges, while the low-mass counterparts are predominantly formed by minor mergers (e.g., Cattaneo et al. 2011; Hopkins et al. 2010). Nevertheless, both simulations and observational studies have shown minor mergers to be more abundant at high masses and more efficient at increasing the mass and size of galaxies than major mergers (e.g., Naab et al. 2007, 2009; Kaviraj et al. 2009, 2013b). Constraining the merger rates and their contribution to galaxy growth requires appropriately discerning between major and minor events, investigating whether disks survive in mergers of disk galaxies, and comparing the observed basic properties with those predicted by simulations of galaxy mergers. Aiming to address these issues, we present a pilot photometric study of a small sample of double nucleus disk galaxies. The sample is drawn from a catalog of 107 minor merger candidates compiled by Gimeno et al. (2004). The candidates were selected through their disk-like morphologies and the lack of strong tidal tails, characteristic of major merger events. Our sample comprises 52 of these systems, all of which have redshifts $z<0.05$ and for which optical images are available in the Sloan Digital Sky Survey Data Release 8. 

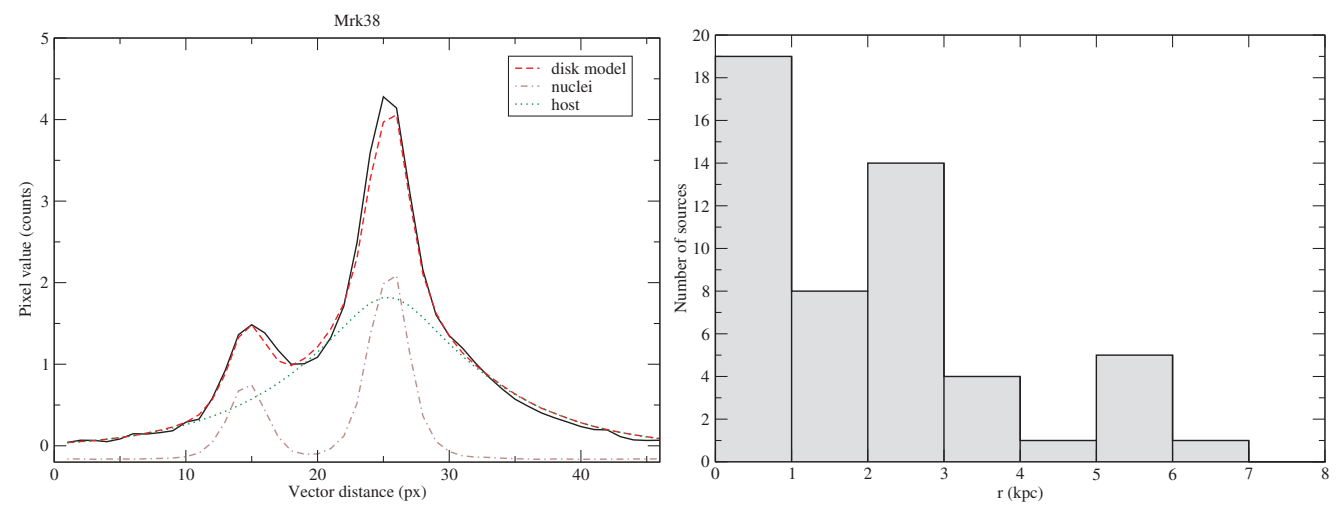

Figure 1. Left: 1D vector extracted along the position of the nuclei of Mrk 38 (solid black line). The imfitfits photometric fit (red dashed line) is shown decomposed into the host fit (green dotted line) and the fit to the two nuclei (brown dotted-dashed line). Right: Distribution of projected separation between the two nuclei.

\section{Analysis}

We use a photometric PSF (Point Spread Function) fitting to estimate the brightness of each of the nuclei of the double nucleus galaxies and the distance between them in the SDSS optical images. A 2D-multicomponent fitting is in a first step attempted using the imfitfits code (McLeod et al. 1998) and a field star image as PSF. We consider two components per merger system: one PSF for each nucleus and one/two exponential disks for the host galaxy/galaxies (Fig. 1, left). This provides satisfactory 2D fittings to $50 \%$ of the sources in our sample. For the remaining 26 sources, a 1D profile is extracted and fitted with the Starlight DIPSO software (Howarth \& Murray 1991). The errors on the intensities of the nuclei and host galaxies and on the relative separation between the nuclei provided by the PSF fittings are $\leqslant 30 \%$ (for details see Mezcua et al., 2014). After correcting for Galactic dust extinction, the intensities are converted to luminosities and the ratio of luminosities $L_{2} / L_{1}$, usually adopted to discern between major and minor merger events (e.g., Lambas et al. 2012), is derived.

\section{Results and discussion}

Adopting a luminosity ratio $>1: 3$ for major mergers, we find that most sources classify as major mergers and that only $38 \%$ (20 out of 52 ) of the systems analyzed qualify as minor mergers. Given their initial visual morphological classification as minor mergers (Gimeno et al. 2004), the major merger systems must be in a post-merger state where the strong optical distortions cannot be any longer recognized.

In the distribution of projected separation between the two nuclei (Fig. 1, right), most sources $(91 \%)$ have projected nuclear separation $\leqslant 4 \mathrm{kpc}$. The number of sources is observed to decrease with nuclear separation, which suggests that these systems are less recognizable as a double nucleus galaxy the greater the distance between the host and the satellite galaxy is. Systems showing the shortest nuclear separation should thus be in a late stage of the merger (i.e., the post-merger state). This is supported by the findings that: 1) $65 \%$ of the sources are fitted by only one galaxy disk instead of two (i.e., the disk of the satellite is no longer recognizable), 2) most sources (62\%) qualify as major mergers, and 3) the main peak of 19 sources observed in Fig. 1 (right) corresponds mostly (15 out of 19$)$ to major merger systems. 

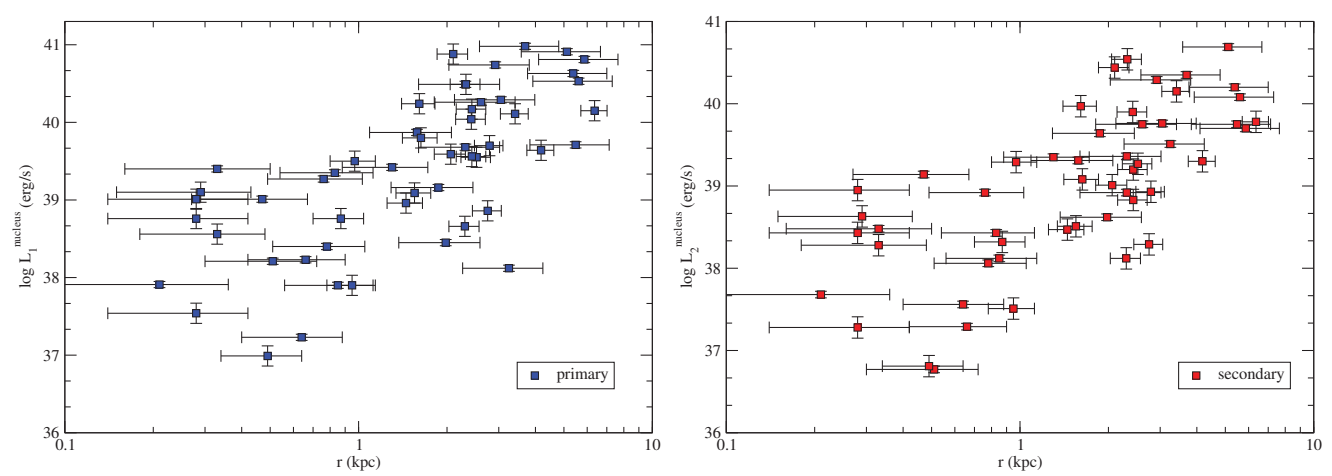

Figure 2. Luminosity of the primary and secondary nucleus versus projected nuclear separation. Adopted from Mezcua et al. (submitted).

\subsection{The nature of the nuclei}

We have shown that the majority of the sources are most plausibly in the post-merger state of a major merger. The presence of an either active (AGN) or dormant black hole in each of the two nuclei is then expected, although the possibility that the secondary nucleus (especially in those sources identified as minor mergers) is related to a stellar cluster or star-forming region cannot be ruled out. High-resolution hard X-ray observations are required to probe the presence of a binary AGN, which has been confirmed for two of the sources in our sample (NGC 5256, Brassington et al. 2007; NGC 3758, Koss et al. 2011). The 19 sources that constitute the main peak in Fig. 1 (right) with projected nuclear distances $\leqslant 1 \mathrm{kpc}$ are of particular interest, as very few $\mathrm{kpc}$ and sub-kpc scale binary AGN have so far been detected (e.g., Komossa et al. 2003; Fabbiano et al. 2011). Of potential interest are also the 20 sources identified as minor mergers, as in these systems the satellite galaxy could host a low-mass black hole (e.g., Bellovary et al. 2010; Mezcua \& Lobanov 2011, Mezcua et al. 2013a,c).

The triggering of AGN activity during a merger event is expected from simulations of disk galaxy mergers (e.g., Hopkins et al. 2008), which also predict the luminosity to decrease with decreasing nuclear separation in the post-merger phase of the merger. This is found for the sources in our sample (Fig. 2), for which a clear correlation between the nuclear luminosity of both the primary and secondary nucleus and the projected nuclear separation is observed. This trend would also be observed if the nuclei were associated to star-formation triggered activity, as the star formation is also expected to drop in the late stages of the merger (i.e., at small nuclear separations).

\section{Summary and conclusions}

The surface brightness profile fitting of a sample of double nucleus disk galaxies has revealed that most of the systems are undergoing a major merger event, contrary to their initial classification as minor mergers, and that they are most plausibly in the post-merger phase of a major merger. This is supported by the finding that most of the sources have nuclear separations $\leqslant 4 \mathrm{kpc}$ and their host galaxies are fitted only by one galaxy disk, and that the luminosity of both nuclei decreases with decreasing nuclear separation as expected from simulations of galaxy mergers. We also identify a sub-sample of 19 systems that could host a sub-kpc binary AGN. 


\section{References}

Bellovary, J. M., Governato, F., Quinn, T. R., et al. 2010, ApJL, 721, L148

Brassington, N. J., Ponman, T. J., \& Read, A. M. 2007, MNRAS, 377, 1439

Cattaneo, A., Mamon, G. A., Warnick, K., \& Knebe, A. 2011, A\& A, 533, A5

Fabbiano, G., Wang, J., Elvis, M., \& Risaliti, G. 2011, Nature, 477, 431

Gimeno, G. N., Díaz, R. J., \& Carranza, G. J. 2004, AJ, 128, 62

Hopkins, P. F., Hernquist, L., Cox, T. J., \& Kereš, D. 2008, ApJS, 175, 356

Hopkins, P. F., Bundy, K., Croton, D., et al. 2010, ApJ, 715, 202

Howarth I. D., Murray J., 1991, SERC-RAL Starlink User Note 50.13

Kaviraj, S., Peirani, S., Khochfar, S., Silk, J., \& Kay, S. 2009, MNRAS, 394, 1713

Kaviraj, S., Cohen, S., Windhorst, R. A., et al. 2013, MNRAS, 429, L40

Komossa, S., Burwitz, V., Hasinger, G., et al. 2003, ApJL, 582, L15

Koss, M., Mushotzky, R., Treister, E., et al. 2011, ApJL, 735, L42

Lambas, D. G., Alonso, S., Mesa, V., \& O'Mill, A. L. 2012, A\&3A, 539, A45

McLeod, B. A., Bernstein, G. M., Rieke, M. J., \& Weedman, D. W. 1998, AJ, 115, 1377

Mezcua, M. \& Lobanov, A. P. 2011, Astronomische Nachrichten, 332, 379

Mezcua, M., Lobanov, A. P., Mediavilla, E., \& Karouzos, M. 2014, ApJ, 784, 16

Mezcua, M., Farrell, S. A., Gladstone, J. C., \& Lobanov, A. P. 2013a, MNRAS, 436, 1546

Mezcua, M., Roberts, T. P., Sutton, A. D., \& Lobanov, A. P. 2013c, MNRAS, 436, 3128

Naab, T., Johansson, P. H., Ostriker, J. P., \& Efstathiou, G. 2007, ApJ, 658, 710

Naab, T., Johansson, P. H., \& Ostriker, J. P. 2009, ApJL, 699, L178 